\title{
Deux dispositifs de contrôle permanent du débit absorbé par une turbine
}

\section{Two continuous-recording instruments for rates of flow absorbed by a turbine}

\author{
PAR \\ R. WOLF \\ $\mathrm{ET}$ \\ P. BLANC-FÉRAUD

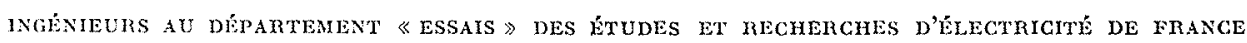

\begin{abstract}
Description de deux dispositifs d̀ réponse linéaire mis au point récemment en vue de l'enregistrement continu du débit turbiné dans quelques usines hydró́lectriques:

$1^{\circ}$ Débitmètre électromagnélique placé en dérivation sur un troncon de la conduite d'amenée de manière à mesurer une fraction connue du débit principal;

$2^{\circ}$ Capteur déprimogìne à transmission électrique et réponse linéaire en fonction du débit. Examen des résultats obtenus. Application a l'enregistrement direct du volume d'eau turbiné.
\end{abstract}

Description of two linear-response instruments recently developed for the continuous recording of rates of flow through turbines at a number of hydro-power installations.

1. A magnetic current meter installed in parallel with a section of the supply duct, in order to measure a known fraction of the main flow; 2. An electrical recording instrument working on the pressure drop principle, giving a linear response to variations in the rate of flow.

Examination of the results obtained. Application of the results to the direct recording of flow rates through turbines.

\section{A. - DISPOSITIF DÉPRIMOGÈNE}

Ce dispositif utilise la chute de pression créée par une singularité du circuit d'alimentation de la turbine, vanne, coude, convergent.

Cette chute de pression est liée au débit par des lois connues pour les systèmes déprimogènes normalisés, mais dans notre cas, l'utilisation d'une singularité quelconque nécessite un étalonnage.

\section{I. - PRINCIPE}

\section{DE LA MESURE ELECTRIQUE}

Nous utilisons un pont de Wheatstone dont l'une des branches comporte deux systèmes de résistance $\left(\mathrm{S}_{1}\right)$ et $\left(\mathrm{S}_{2}\right)$, les trois autres branches étant des résistances fixes.
Le système $\left(S_{1}\right)$ est fonction de la grandeur à mesurer, c'est-à-dire, dans notre cas, du débit Q. On réalise un asservissement à partir du courant de déséquilibre qui rééquilibre le pont en agissant sur le système $\left(\mathrm{S}_{2}\right)$.

On verra que, si la variation du système $\left(S_{1}\right)$ qui déséquilibre le pont est proportionnelle au carré du débit, la variation du système $\left(S_{2}\right)$ nécessaire au rééquilibrage est proportionnelle aul débit.

Le système $\left(S_{1}\right)$ est un manomètre différentiel Beaudouin à membrane et potentiomètre incorporés.

Le système $\left(\mathrm{S}_{2}\right)$ est constitué de deux potentiomètres rigoureusement proportionnels $S_{m}$ et $k \mathrm{~S}_{m}$ dont les curseurs sont mécaniquement soli- 


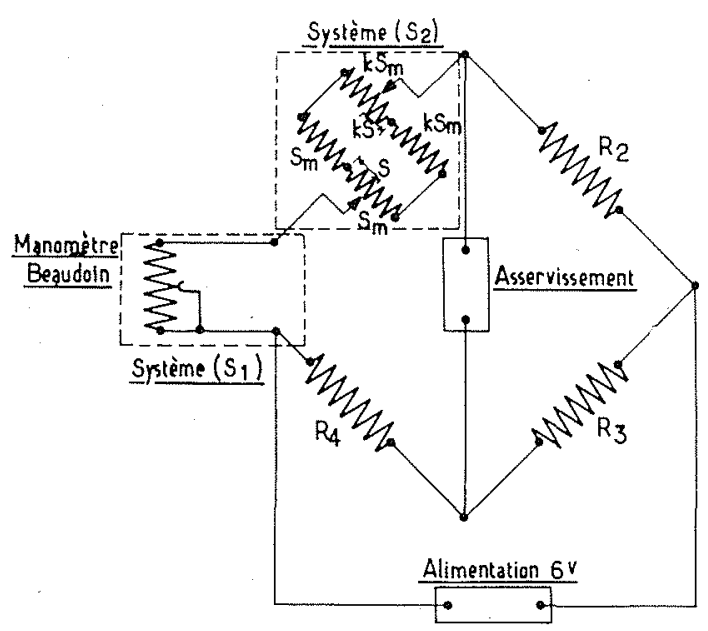

FIG. 1

Schéma de princinc du débitmètre.

daires et de deux résistances fixes respectivement égales à $S_{m}$ et $k S_{m}$.

L’ensemble du pont est représenté sur la figure 1 .

La perte de charge utilisée est proportionnelle au carré du débit et l'équation du manomètre différentiel s'écrit :

$$
\mathrm{R}=\mathbf{R}_{0}+\left(\mathbf{R}_{m}-\mathbf{R}_{0}\right)\left(\frac{q}{q_{m}}\right)^{2}
$$

$\mathrm{R}_{0}$ résistance à débit nul;

$\mathrm{R}_{m}$ résistance au débit maximal.

Soit \& la résistance équivalente au système $\left(\mathrm{S}_{2}\right)$ :

$$
\bullet=\frac{S_{m}^{2}(1+k)^{2}-S^{2}(1-k)^{2}}{2 S_{m}(1+k)}
$$

L'impédance 'T de la branche s'écrit :

$$
\begin{aligned}
\mathrm{T}=\mathrm{R}_{0} & +\left(\mathrm{R}_{m}-\mathrm{R}_{0}\right) \cdot\left(\frac{q}{q_{m}}\right)^{2} \\
& +\frac{\mathrm{S}_{m}{ }^{2}(1+k)^{2}-\mathrm{S}^{2}(1-k)^{2}}{2 \mathrm{~S}_{m}(1+k)}
\end{aligned}
$$

relation de la forme $\mathrm{A} q^{2}+\mathrm{BS}^{2}+\mathrm{C}=0$, qui est l'équation d'une conique en axes $q$, S. Celle-ci se décomposera en deux droites passant par l'origine $\mathrm{si}$, et si seulement $\mathrm{S}=0$ pour $q=0$. Nous fixons la pente de la droite en imposant la condition $\mathrm{S}=\mathrm{S}_{m}$ pour $q=q_{m}$.

L'équation de la droite de réponse est alors déterminée; c'est :

$$
\mathrm{S}=\frac{\mathrm{S}_{m}}{q_{m}} q
$$

L'asservissement est conçu pour que l'impédance de la branche de mesure soit constante en fonction du débit.

Ce qui impose :

$$
\mathrm{R}_{m}-\mathrm{R}_{0}=\mathrm{S}_{m} \frac{(1-k)^{2}}{2(1+k)}
$$

La valeur de $\mathrm{T}$ devient :

$$
\mathrm{T}=\mathrm{R}_{0}+\frac{\mathrm{S}_{m}(1+k)}{2}
$$

l'équation (5) n'a qu'une seule racine en $S_{m}$. Cette racine est fonction des données du manomètre par $R_{m}$ et $R_{0}$ et du choix des potentiomètres du système $\left(S_{2}\right)$ par $k$.

La valeur de ' $\mathrm{T}$ est déterminée lorsqu'on a calculé $\mathrm{S}_{m}$.

Il est évident, d'après l'équation (5), que ce pont fonctionnerait pour un manomètre et une perte de charge correspondant au débit maximal, c'est-à-dire à $R_{m}-R_{0}$ donnés. Chaque fois qu'on changerait l'un des facteurs, il faudrait changer les potentiomètres $\mathrm{S}_{m}$ et $k \mathrm{~S}_{m}$. Deux résistances de réglage supplémentaires sont représentées sur la figure 2 . Nous verrons plus loin que leur présence ne modifie pas la linéarité de la réponse. Elles permettront d'adapter le pont à n'importe quelle valeur de $R_{m}-R_{0}$.

Reprenons les calculs précédents en tenant compte des résistances de réglage.

On trouve:

$$
\begin{aligned}
= & =\frac{k+1}{2} \mathrm{~S}_{m} \\
& -\mathrm{S}^{2}\left[\frac{1}{\left(\frac{k+1}{2 k}\right)^{2}+\frac{k+1}{2 k} \mathrm{~S}_{m}}+\frac{(k-1)^{2}}{2(k+1) \mathrm{S}_{m}}\right]
\end{aligned}
$$

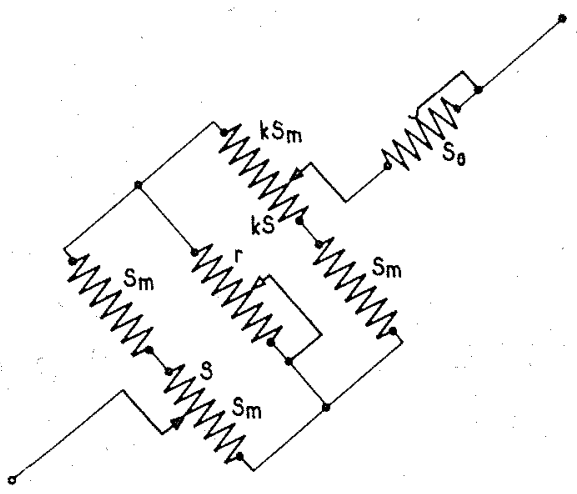

Fyg. 2

Système ( $\mathrm{s}$ ) modifié. 


$$
\begin{aligned}
& \mathrm{T}=\mathrm{R}_{0}+\left(\mathrm{R}_{m}-\mathrm{R}_{0}\right)\left(\frac{\mathrm{S}_{m}}{q_{m}}\right)^{2} \\
& -\mathrm{S}^{2}\left[\frac{1}{\left(\frac{k+1}{2 k}\right)^{2} r+\frac{k+1}{2 k} \mathrm{~S}_{m}}+\frac{(k-1)^{2}}{2(k+1)^{2}}\right] \\
& \quad+\frac{k+1}{2} \mathrm{~S}_{m}+\mathrm{S}_{0}
\end{aligned}
$$

En $\mathrm{y}$ faisant :

$$
r=\infty \quad \text { et } \mathrm{S}_{0}=0
$$

on retrouve la relation (6). $\mathrm{Si}$, à débit nul $\mathrm{S}=0$ et au débit maximal, $S=S_{m}$ la condition pour que $\mathrm{T}$. soit constant devient :

$\mathrm{R}_{m}-\mathrm{R}_{0}=\frac{2}{\left(\frac{k+1}{2 k}\right)^{2} \times r+\frac{k+1}{2 k} \mathrm{~S}_{m}}+\frac{(k-1)^{2} \mathrm{~S}_{m}}{2(k+1)}$

analogue à l'équation (5).

Cette égalité étant satisfaite, $T$ est indépendant de $q$ et vaut :

$$
\mathrm{T}=\mathrm{R}_{0}+\mathrm{S}_{0}+\frac{k+1}{2} \mathrm{~S}_{m}
$$

Les deux potentiomètres $\mathrm{S}_{m}$ et $k \mathrm{~S}_{i l}$ seront pris égaux, c'est-à-dire que $1 \leqslant k \leqslant 1,03$; dans ces conditions nous pouvons négliger le terme :

$$
\left[(k-1)^{2} / 2 \mathrm{~S}_{m}(k+1)\right]
$$

dans l'équation (8).

Les valeurs numériques du pont seront calculées d'après la résolution du système d'équations générales, compte tenu du fait que nous devons éliminer les solutions négatives.

En particulier, $r>0$ et $S_{0}>0$ entraînent:

$$
\mathrm{S}_{m} \geqslant \mathrm{R}_{m}-\mathrm{R}_{0} \quad \text { et } \mathrm{T} \geqslant \mathrm{R}_{0}+\frac{k+1}{2 k} \mathrm{~S}_{m}
$$

\section{Remarque :}

Protection différentielle des conduites forcées.

Il s'agit d'assurer la fermeture automatique de la vanne de tête des conduites en cas d'avarie de ces dernières. Pour cela, nous comparons en permanence les débits à chacune des extrémités de la conduite à l'aide de deux débitmètres du type de celui que nous venons de décrire.

Chacun des débitmètres a $\mathrm{S}=\left(\mathrm{S}_{m} / q_{m}\right) q$ comme droite de réponse; $q_{m}$ est forcément le même et la résistance $r$ permet d'avoir aussi le même $S_{m}$ pour chaque débitmètre. Les deux droites sont alors confondues, et on peut utiliser un seul asservissement pour les deux ponts, la même

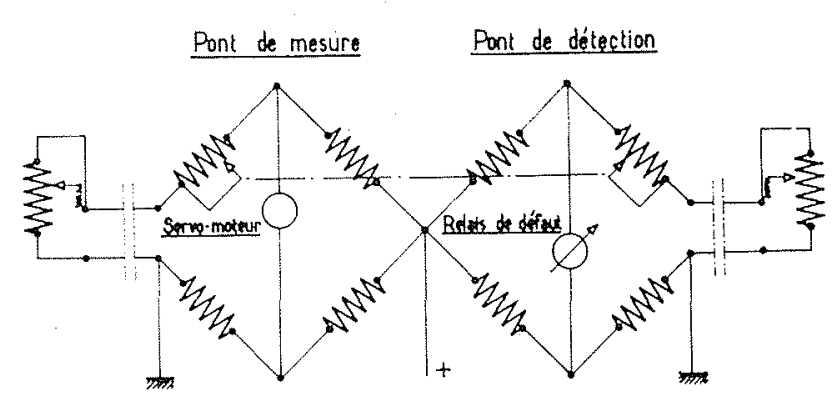

Fic. 3

Schéma de principe

de la protection différentielle.

variation de $q$ entrainant la mème variation de $\mathrm{S}$.

Tout écart de débit entre les deux sections se traduit alors par une variation de l'intensité $i$ dans la diagonale intérieure du pont de détection, intensité de commande du relais de signal de défaut (fig. 3).

\section{II. - REALISATION}

\section{II.1. Choix de l'emplacement des capteurs.}

Il s'agit d'utiliser une différence d'altitude piézométrique constante dans le temps. Cette condition nous a amenés à utiliser une singularité de l'installation dans laquelle les pertes de charges interviennent relativement peu, car, généralement variables dans le temps, elles risquent d'entraîner une infidélité du contrôle des débits.

La singularité que nous avons choisie à l'usine de Serre-Ponçon est constituée par les deux convergents d'entrée et de sortie d'une vanne papillon (fig. 4).

Pour le débit maximal, nous disposons d'une différence de hauteur piézométrique de $1,50 \mathrm{~m}$.

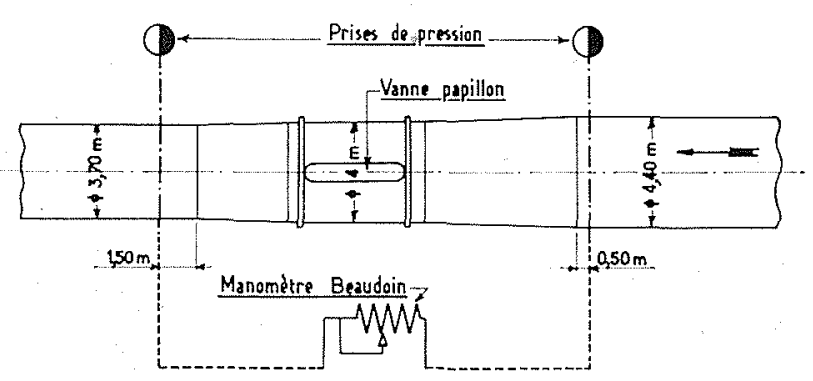

FIQ. 4

Schéma d'installation du capteur. 
Cette dénivellation est due en grande partie à l'accroissement d'énergie cinétique de l'eau au détriment de son énergie de pression au passage du convergent.

\section{II.2. Description de l'appareillage.}

Le capteur de pression différentiel des Ets Beaudouin comporte un potentiomètre incorporé de 1500 ohms de résistance; chaque spire correspond à une résistance de 5 ohms environ. La fidélité des contacts est restée bonne pendant plus d'un an de fonctionnement permanent; il semble que le contact soit surtout sensible aux déplacements très rapides.

Le pont de mesure comporte un ensemble de quatre résistances de $2900 \mathrm{ohms}$ environ et de deux résistances de réglage de 200 et 2500 ohms.

La cellule de linéarisation de la réponse est constituée par deux résistances linéaires de $2700 \mathrm{ohms}$ environ, d'une longueur d'enroulement de $260 \mathrm{~mm}$ pour un pas de $0,2 \mathrm{~mm}$, la linéarité est d'environ $0,2 \%$; l'alignement entre elles de ces deux résistances est aussi de l'ordre de $0,2 \%$. Les curseurs qui les parcourent sont montés sur un même chariot actionné par une chaîne solidaire d'un moteur d'entraînement.

La chaîne d'asservissement comprend un amplificateur, un servo-moteur auquel est couplée une génératrice tachymétrique qui fournit à l'amplificateur une tension appliquée en contreréaction sur la tension d'entrée.

La tension continue de déséquilibre qui appa- raît aux bornes du pont et qui correspond à une variation du débit est convertie à l'aide d'un vibreur en une tension alternative qui est amplifiée et commande le servo-moteur.

\section{II.3. Mise en œuvre.}

\section{EQuilibrage du pont :}

Pour satisfaire les conditions de linéarisation, dans un premier temps, à débit nul, le curseur de la résistance de mesure sur la position zéro, on règle la résistance $S_{0}$ pour que l'intensité du courant dans la diagonale du pont soit nulle.

Dans le deuxième temps, le débit étant voisin du débit maximal $q_{m}$ et le curseur de la résistance de lecture sur la position $\mathrm{S}_{m}$, on annule le courant dans la diagonale du pont en jouant sur $r$.

\section{Protectron des capteurs :}

Il faut protéger les capteurs pour ne pas détériorer leur membrane, par exemple lorsqu'on ferme la vanne papillon. En effet, dans ce cas, il y aurait d'un côté la pression de la conduite, de l'autre la pression atmosphérique ou du moins une pression très faible, et on claquerait la membrane.

Pour éviter cet ennui, on «by-passe» le capteur lorsque la vanne papillon se ferme grâce à une électrovanne commandée par le servomoteur de la vanne papillon.

\section{III. - RESULTATS}

\section{III.1. Fonctionnement de l'appareil.}

Quatre débitmètres de ce type ont été installés à Serre-Ponçon sur chacun des groupes et nous ont donné entière satisfaction après un fonctionnement continu de six mois. La graduation de l'échelle avait été réalisée en calculant le débit $q_{m}$ d'après la puissance. Lors de la mise en ouvre, nous avons constaté un léger pompage hydraulique que nous avons supprimé en étranglant les manomètres à l'aide des robinets à pointeau. Le réglage est fidèle dans le temps, mais assez délicat.

\section{III.2. Précision de la mesure.}

Les équations qui régissent la mesure sont les suivantes :

$$
\mathrm{A}\left\{\begin{array}{cl}
\mathrm{S}=\frac{\mathrm{S}_{m}}{q_{m}} q & \left\{\begin{array}{l}
\text { réponse linéaire } \\
\mathrm{T}=\mathrm{R}_{0}+\mathrm{S}_{0}+\frac{k+1}{2} \mathrm{~S}_{m}
\end{array}\right. \\
\mathrm{R}_{m}-\mathrm{R}_{0}=\frac{\mathrm{S}=0}{\left(\frac{k+1}{2 k}\right)^{2} \frac{\mathrm{S}_{m}{ }^{2}}{\frac{k+1}{2} \mathrm{~S}_{m}}+\frac{(k-1)^{2}}{2(k+1)} \mathrm{S}_{m}} & \begin{array}{l}
\mathrm{S}=\mathrm{S}_{m} \\
q=q_{m}
\end{array} \text { conditions limites }
\end{array}\right.
$$


Pour le calcul d'erreur, nous prendrons $k=1$ et le système (A) différencié s'écrit :

$$
\left\{\begin{aligned}
\frac{\Delta \mathrm{S}}{\mathrm{S}} & =\frac{\Delta \mathrm{S}_{m}}{\mathrm{~S}_{m}}-\frac{\Delta q_{m}}{q_{m}}+\frac{\Delta q}{q} \\
\Delta \mathrm{T} & =\Delta \mathrm{R}_{0}+\Delta \mathrm{S}_{0}+\Delta \mathrm{S}_{m} \\
\frac{\Delta\left(\mathrm{R}_{m}-\mathrm{R}_{0}\right)}{\mathrm{R}_{m}-\mathrm{R}_{0}} & =2 \frac{\Delta \mathrm{S}_{m}}{\mathrm{~S}_{m}}-\frac{\Delta r}{r+\mathrm{S}_{m}}-\frac{\Delta \mathrm{S}_{m}}{r+\mathrm{S}_{m}} \\
\Delta \mathrm{R} & =\Delta \mathrm{R}_{0}+\left(\mathrm{R}_{m}-\mathrm{R}_{0}\right)\left(\frac{q}{q_{m}}\right)^{2}\left[\frac{\Delta\left(\mathrm{R}_{m}-\mathrm{R}_{0}\right)}{\mathrm{R}_{m}-\mathrm{R}_{0}}+2 \frac{\Delta q}{q}-2 \frac{\Delta q_{m}}{q_{m}}\right]
\end{aligned}\right.
$$

Nous avons là un système linéaire de quatre équations à dix inconnues. On peut donc éliminer trois inconnues et il nous restera une relation entre les sept inconnues restantes. Nous avons conservé comme inconnues :

$\Delta q$ erreur sur le débit mesuré;

$\Delta \mathrm{S}$ erreur sur la résistance de lecture;

$\Delta r$ et $\Delta \mathrm{S}_{0}$ caractérisant les réglages, c'està-dire la linéarité de la réponse et la position de la droite de réponse;

$\Delta \mathrm{T}$ sensibilité du pont de mesure;

$\Delta \mathrm{R}$ sensibilité du capteur;

$\Delta q_{m}$ erreur sur l'étalonnage.

Après élimination des trois inconnues, compte temu des équations de départ et des valeurs choisies, nous obtenons :

$$
\begin{aligned}
& {\left[\frac{\Delta q}{q}-\frac{\Delta q_{m}}{q_{m}}\right]\left[\left(\frac{q_{m}}{q}\right)^{2}+1\right]} \\
& =\frac{\Delta \mathrm{S}}{\mathrm{S}_{m}} \frac{q_{m}}{q}\left[\left(\frac{q_{m}}{q}\right)^{2}-1\right]+\frac{\Delta \mathrm{R}}{\mathrm{R}_{m}-\mathrm{R}_{0}}\left(\frac{q_{m}}{q}\right)^{2} \\
& \quad+\left(\Delta \mathrm{S}_{0}+\Delta \mathrm{T}\right) \frac{1}{\mathrm{R}_{m}-\mathrm{R}_{0}}\left(\frac{q_{m}}{q}\right)^{2}+\frac{\Delta r}{\mathrm{~S}_{m}}
\end{aligned}
$$

$\Delta \mathrm{T}$ est définie comme la plus petite variation d'impédance de la branche contenant la cellule qui se traduise par un déplacement du curseur. D'après les caractéristiques du pont, l'asservissement réagit pour une variation de courant dans la diagonale :

$$
\Delta i=\frac{2,5 \cdot 10^{-6}}{10^{3}}=2,5 \cdot 10^{-9} \mathrm{~A} .
$$

Il est alors facile de calculer la valeur de $\Delta \mathrm{T}$ correspondant à $\Delta i$. Le calcul donne :

$$
\Delta \mathrm{T}=0,0185 \Omega
$$

$\mathrm{S}_{0}$ est la résistance de réglage qui permet d'équilibrer le pont lorsque $S=0$ et $R=R_{0}$.

Le calcul est le même que celui qui a été fait pour $\Delta \mathrm{T}$ en tenant compte que dans la diagonale du pont, nous avions un galvanomètre A.O.I.P. type G.122 au lieu d'avoir l'amplifica- teur de l'asservissement. La sensibilité de ce galvanomètre est de $1,3 \cdot 10^{-8} \mathrm{~A}$ et sa résistance interne $120 \Omega$.

Le calcul nous a donné : $\Delta \mathbf{S}_{0}=0,116$.

$\Delta r$ est la résistance de réglage qui permet d'avoir un courant nul dans la diagonale du pont lorsque $\mathrm{S}=\mathrm{S}_{m}$ et $q=q_{m}$, e'est-à-dire la résistance qui permet de réaliser l'égalité.

$$
\mathrm{R}_{m}-\mathrm{R}_{0}=\frac{\mathrm{S}_{m}^{2}}{\left(\frac{k+1}{2 k}\right)^{2} r+\frac{k+1}{2 k} \mathrm{~S}_{m}}
$$

$\Delta r$ est relié à l'impédance totale de la branche du pont qui le contient par la formule :

$$
\mathrm{T}=\mathrm{S}_{0}+\mathrm{R}+\mathrm{S}_{m}-\frac{\mathrm{S}^{2}}{r+\mathrm{S}_{m}}
$$

Autrement dit :

$$
\frac{\Delta^{\prime} \mathrm{T}}{\mathrm{T}}=\frac{\Delta r}{r+\mathrm{S}_{m}}
$$

d'où $\Delta r=0,116 \times \frac{500+2700}{2900}=0,20 \Omega$

car nous avons déjà vu que le galvanomètre A.O.I.P. utilisé pour les réglages décelait une variation de résistance d'une branche de $0,116 \Omega$. $\Delta S$ est l'erreur de lecture.

$\mathrm{S}_{m}$ est un potentiomètre de $2700 \Omega$ de $260 \mathrm{~mm}$ de long et de pas $0,2 \mathrm{~mm}$.

Or, à la lecture, on ne peut pas apprécier mieux que le demi-millimètre, par suite des jeux mécaniques des différents organes d'asservissement et d'enregistrement.

Nous avons done :

$$
\Delta S=\frac{2700}{1300} \times 2,5=5,2 \Omega
$$

Enfin $\Delta R$, résistance d'une spire du potentiomètre du capteur manométrique, est égale à $5 \Omega$.

Si nous remarquons que :

$$
\frac{\Delta q}{q}-\frac{\Delta q_{m}}{q_{m}}=\frac{\Delta q / q_{m}}{q / q_{m}}
$$

nous pouvons passer en variables adimension- 


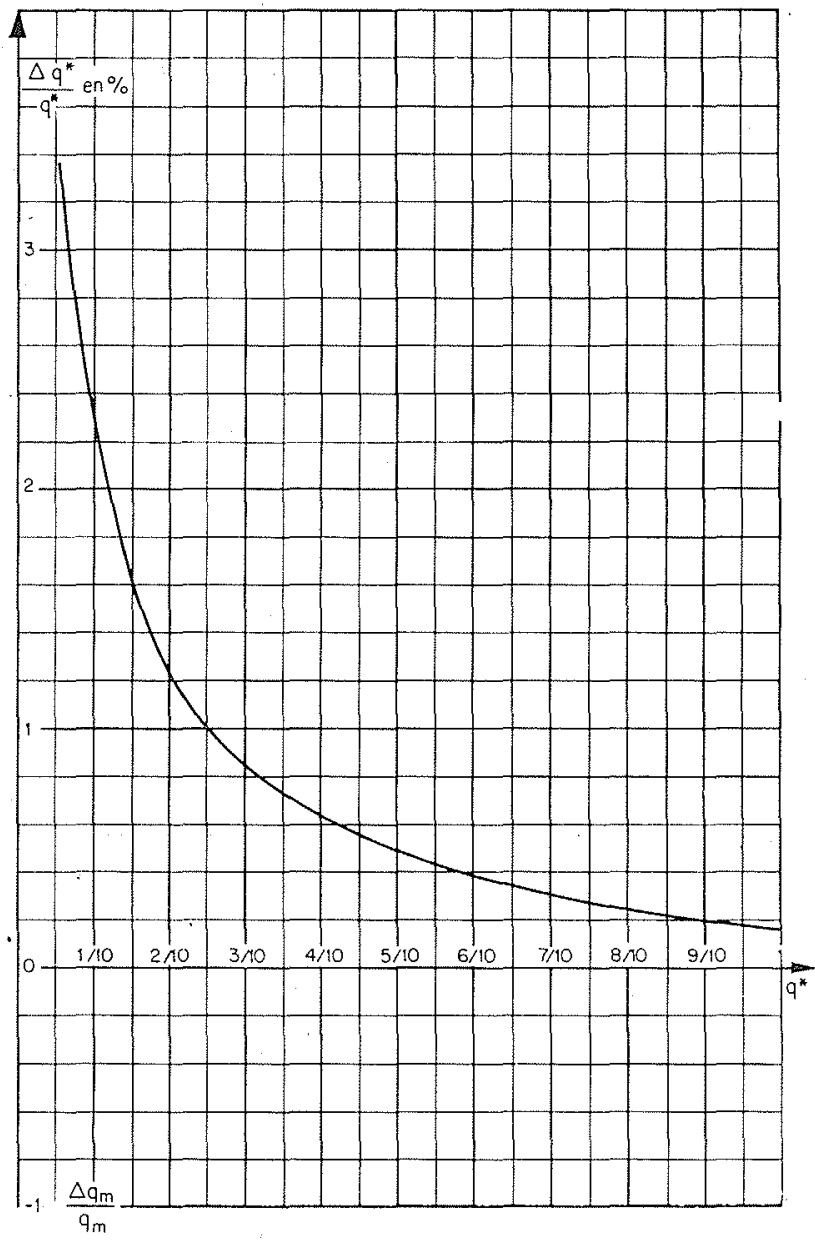

Fici. 5

Courbe de precision. nelles en posant $\left(q / q_{m}\right)=q^{*}$ et nous aboutissons à l'équation :

$\frac{\Delta q^{\star}}{q^{\star}}=$

$\frac{0,74 \cdot 10^{-4} q^{*}-2 \cdot 10^{-3} q^{\star 2}+3,2 \cdot 10^{-5} q^{*}+2 \cdot 10^{-3}}{q^{\star}\left(1+q^{* 2}\right)}$

La courbe est représentée sur la figure 5.

\section{IV. - TOTALISATION DES DÉBITS}

Une résistance de $2700 \mathrm{ohms}$ est calée avec les deux résistances de mesure. Elle est alimentée par une tension stabilisée de 60 volts environ. Un diviseur potentiométrique de grande résistance recueille Ia tension proportionnelle au débit avec une déformation inférieure à $1 \%$ (on a pris 200000 ohms). Cette tension, qui varie de 0 à 6 volts pour toute la gamme de mesure, pilote la grille d'une triode $\mathrm{T}_{1}$. Une seconde triode, $T_{2}$, recoit une tension variable.

Le moteur totalisateur est branché entre les cathodes des triodes $\mathrm{T}_{1}$ et $\mathrm{T}_{2}$. Il est soumis à la différence des tensions qui apparaissent entre les cathodes. Un tel montage, extrêmement simple, permet de conserver la linéarité de la réponse et d'éviter les déformations dues $\dot{a}$ de faibles impédances dans le circuit mesure.

L'enroulement tachymétrique d'un tel moteur permet de disposer d'une tension stable que l'on peut renvoyer sur un indicateur à aiguille. Le moteur entraine un totalisateur mécanique.

\section{B. - DISPOSITIF ÉLECTROMAGNÉTIQUE}

\section{I. - PRINCIPE DE LA MÉTHODE}

Tout conducteur se déplaçant dans un champ magnétique est le siège d'une f.e.m. induite $e$. Si nous considérons un liquide s'écoulant dans une conduite isolante circulaire, soumis à un champ magnétique uniforme perpendiculaire à l'axe de la conduite, on recueille aux extrémités d'un diamètre pérpendiculaire au champ magnétique une tension $e$.

La théorie montre que cette tension est indépendante de la résistivité de l'eau et rigoureusement proportionnelle à la distance entre électrodes, à l'induction magnétique et à la vitesse moyenne dans la section de mesure, quelle que soit la nature de l'écoulement.
Il est possible de disposer une tuyère électromagnétique dans une conduite forcée. Cette solution présente l'inconvénient d'avoir à vidanger Ia conduite forcée toutes les fois que l'on veut accéder au capteur. En outre, la présence de la tuyère au sein de l'écoulement modifie la répartition des vitesses dans la section de mesure et il faut quand même procéder par étalonnage.

Il est préférable d'installer, quand cela est possible, un débitmètre en dérivation sur la conduite forcée.

\section{Remarque :}

La Division Technique Générale d'Electricité de France a étudié à l'usine de La Bridoire, le comportement des compteurs d'eau dans une 


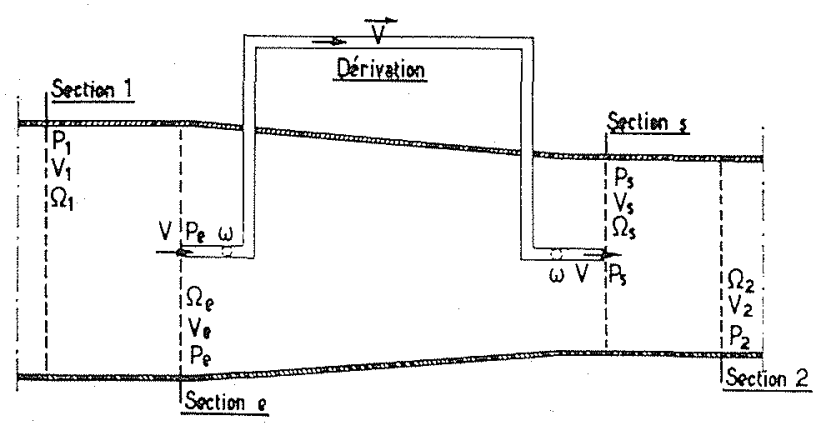

FIG. 6

Schéma de principe.

dérivation, et a conclu que ce système n'ćtait pas assez sûr lorsqu'on utilise les compteurs dans une eau qui n'a pas été parfaitement filtrée, d'où l'idée d'y installer un débitmètre électromagnétique.

II nous faut définir la relation existant entre le débit dérivé $q$ et le débit principal $Q$.

Il existe un cas qui peut ètre résolu par le calcul : celui qui est schématisé sur la figure 6 . Les hypotheses dans ce cas sont les suivantes:

- L'entrée de la dérivation est constituée par une prise dynamique analogue à celle d'un Pitot;

- Dans la section de la conduite à l'aval immédiat de la fin de la dérivation, les filets sont rectilignes et parallèles.

Entre les sections (1) et (S) on peut appliquer Ie théorème de Bernouilli, tandis qu'entre les sections (S) et (2), nous sommes contraints d'appliquer le théorème des quantités de mouvement. Le calcul conduit à l'équation suivante :

$$
\begin{array}{r}
\frac{\mathrm{Q}^{2}}{2 g}\left[\frac{1}{\Omega_{1}^{2}}+\frac{2}{\left(\Omega_{2}-\omega\right) \Omega_{2}^{2}}\right]-\frac{4 Q q}{2 g} \cdot \frac{1}{\Omega_{2}\left(\Omega_{2}-(1)\right)} \\
\quad+\frac{2 q^{2}}{2 g} \cdot \frac{1}{\Omega_{2}\left(\Omega_{2}-\omega\right)}=\xi_{e s}-\left[\frac{\mathrm{P}_{1}}{\bar{\omega}}-\frac{\mathrm{P}_{2}}{\bar{\omega}}\right]
\end{array}
$$

C'est l'équation d'une conique, qui se décompose en deux droites si la perte de charge dans la dérivation el la différence de hauteur piézométrique au niveau des branchements sont quadratiques en fonction du débit.

Etudions le cas d'un convergent :

$$
\frac{\mathrm{P}_{1}-\mathrm{P}_{2}}{\omega}=\frac{\mathrm{V}_{2}^{2}-\mathrm{V}_{1}^{2}}{2 g}+\xi_{12} .
$$

$\xi_{12}$ est en général négligeable devant la variation d'énergie cinétique.
L'équation de la conique décomposée devient :

$$
\begin{aligned}
\mathrm{Q}^{2}\left[1+\frac{2 \alpha}{1-\alpha}\right]- & 4 \mathrm{Q} q \frac{1}{1-\alpha} \\
& +2 q^{2}\left(\frac{1}{1-\alpha}-\frac{k}{\alpha^{2}}\right)=0
\end{aligned}
$$

avec la notation $\alpha=\frac{(1)}{\Omega_{2}}$

$$
\text { Seules les solutions } q \neq \mathbf{0} \quad \mathbf{Q} \neq 0 \text { sont }
$$
intéressantes.

Les solutions de l'équation du deuxième degré en $(\Omega / q)$ a deux racines de signe contraire et seule la racine $\neq 0$ possede une signification physique.

On trouve :

$$
\frac{Q}{q}=\frac{2}{1+\alpha}\left\lceil 1+\frac{1}{\alpha} \sqrt{\frac{\alpha^{2}+k\left(1-\alpha^{2}\right)}{2}}\right\rfloor
$$

$Q$ est proportionnel à $q$.

Revenons au cas de l'usine de La Bridoire (fig. 7). Nous avons pensé, par analogie avec le calcul qui vient d'être fait, que si les pertes de charge dans la dérivation étaient quadratiques ainsi que la différence de hauteur piézométrique dans la conduite à hauteur des branchements, on pourrait espérer une réponse linéaire. Comme nous l'avons déjà souligné, le calcul théorique de la loi entre le débit principal et le débit dérivé est très complexe dans. le cas général, car nous ignorons tout des phénomènes hydrauliques qui se situent aux niveaux des branchements, y compris le fait de savoir si la perte de charge qui s'y trouve est quadratique ou pas. De toutes facons, il est probable qu'en utilisant une dérivation ayant des pertes de charge quadratiques importantes, on puisse négliger les pertes de charge singulières des branchements devant ces dernières, ce qui nous conduit à une réponse linéaire.

Notons que, d'après le calcul théorique, le coeflicient de proportionnalité entre $Q$ et $q$ dépend de deux paramètres $\alpha$ et $k$.

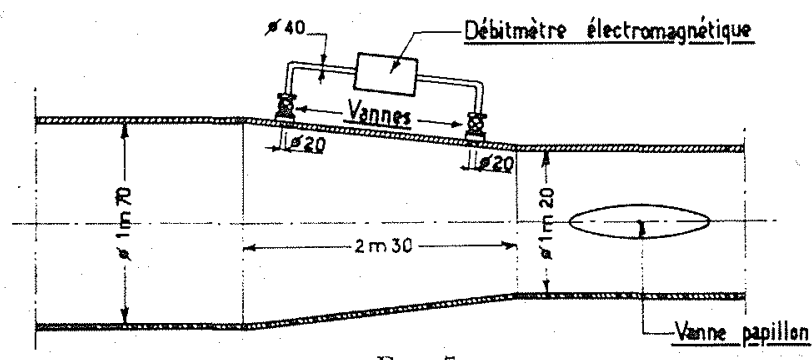

Fia. 7

Schéma de la dérivation a l'usine de lat Bridoire. 
$\alpha$ est une constante de l'installation;

$k$ dépend des pertes de charge quadratiques de la dérivation.

La fidélité du dispositif est caractérisée par la constante de ce coefficient.

La différence de hauteur piézométrique dans la conduite est aussi quadratique, pour deux raisons :

- D'une part, le régime est turbulent; en effet le nombre de Reynolds varie de 6000000 à 750000 lorsque le débit varie de $8 \mathrm{~m}^{3} / \mathrm{s}$ à $1 \mathrm{~m}^{3} / \mathrm{s}$. On peut donc considérer que les pertes de charge sont proportionnelles au débit.

- D'autre part, la différence de pression due à l'accroissement d'énergie cinétique de l'eau dans le convergent est, elle aussi, quadratique.

\section{I1. - REALISATION}

\section{II.I. Description de l'appareillage.}

Le dispositif de mesure comporte essentiellement un électro-aimant magnétisé par un enroulement inducteur alimenté en courant alternatif $50 \mathrm{~Hz}$ et un tube isolant dans lequel l'eau s'écoule: ce tube cylindrique, de diamètre égal à celui de la dérivation soit quatre centimètres, porte deux électrodes de platine serties sur un diamètre perpendiculaire $\dot{a}$ la direction du champ magnétique.

Les bobines inductrices sont alimentées en parallèle avec l'ensemble électronique qui amplifie et mesure la différence de potentiel induite. Ces deux ensembles sont régulés en intensité par une lampe fer-hydrogène qui permet ainsi de libérer des variations lentes du réseau. Le schéma de principe est représenté sur la figure 8 .

La tension d'entrée est, pour le point nominal,

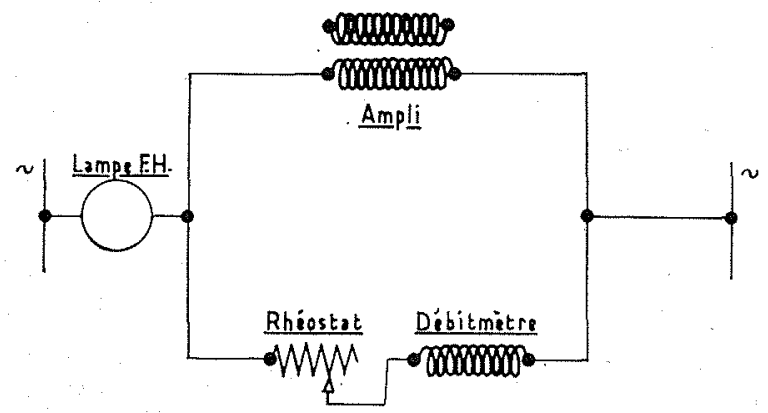

FIG. 8

Schéma de principe. de l'ordre de 1 millivolt. Le gain de l'ensemble électronique est de l'ordre de 1500 .

L'entrée de l'amplificateur est un transformateur de mesure qui permet de réaliser, sans point commun avec la tuyère, d'une part la compensation de la tension statique, d'autre part la vérification du gain de l'amplificateur.

\section{CoMpensation :}

La tension de compensation, variable en phase et en amplitude, est obtenue à partir de la tension d'alimentation filament à travers un pont résistance-capacité, et envoyée à un enroulement du transformateur d'entrée. Ainsi la compensation reste, à très peu de choses près, valable quand le courant varie dans l'alimentation du débitmètre.

\section{TENSION ÉTALON :}

Il s'agit en fait d'une vérification de gain par l'intermédiaire d'une tension de référence qu'on envoie à l'entrée de l'amplificateur après avoir éliminé la tension tuyère à l'aide d'un commutateur. A cette tension correspond une déviation de l'enregistreur que nous avons choisi de l'ordre de grandeur du débit nominal, l'amplificateur travaillant ainsi dans les mêmes conditions de charge. Nous avons ainsi pu vérifier que les dérivées de gain sont négligeables.

\section{II.2. Etalonnage, mise en cuvre.}

II s'agit de déterminer empiriquement la loi :

$$
q=f(\mathrm{Q}) \text {. }
$$

Le circuit dérivé a d'abord été étudié en laboratoire. Nous avons relevé les indications du débitmètre et la différence de hauteur piézométrique aux bornes de la dérivation en fonction du débit $q$ qui la traverse. Puis la dérivation a été montée sur la conduite forcée.

$q$ était connu d'après les courbes obtenues en laboratoire de deux manières différentes: soit d'après les indications du débitmètre, soit d'après contrôle piézométrique.

$Q$ nous était fourni d'après un contrôle piézométrique sur le convergent.

Nous avons alors tracé les deux courbes suivantes:

- $q$ d'après contrôle piézométrique $=f(\mathrm{Q})$.

Nous avons obtenu la droite :

$$
q \mathrm{~m}^{3} / \mathrm{s}=0,14 \cdot 10^{-3} \mathrm{Q} \mathrm{m}^{3} / \mathrm{s} \text { (fig. 9) }
$$

- $q$ d'après indication $\mathrm{du}$ débitmètre $=f(\mathrm{Q})$.

$$
q \mathrm{~m}^{3} / \mathrm{s}=0,14 \cdot 10^{-3} \mathrm{Q} \mathrm{m}^{3} / \mathrm{s} \text { (fig. 10) }
$$




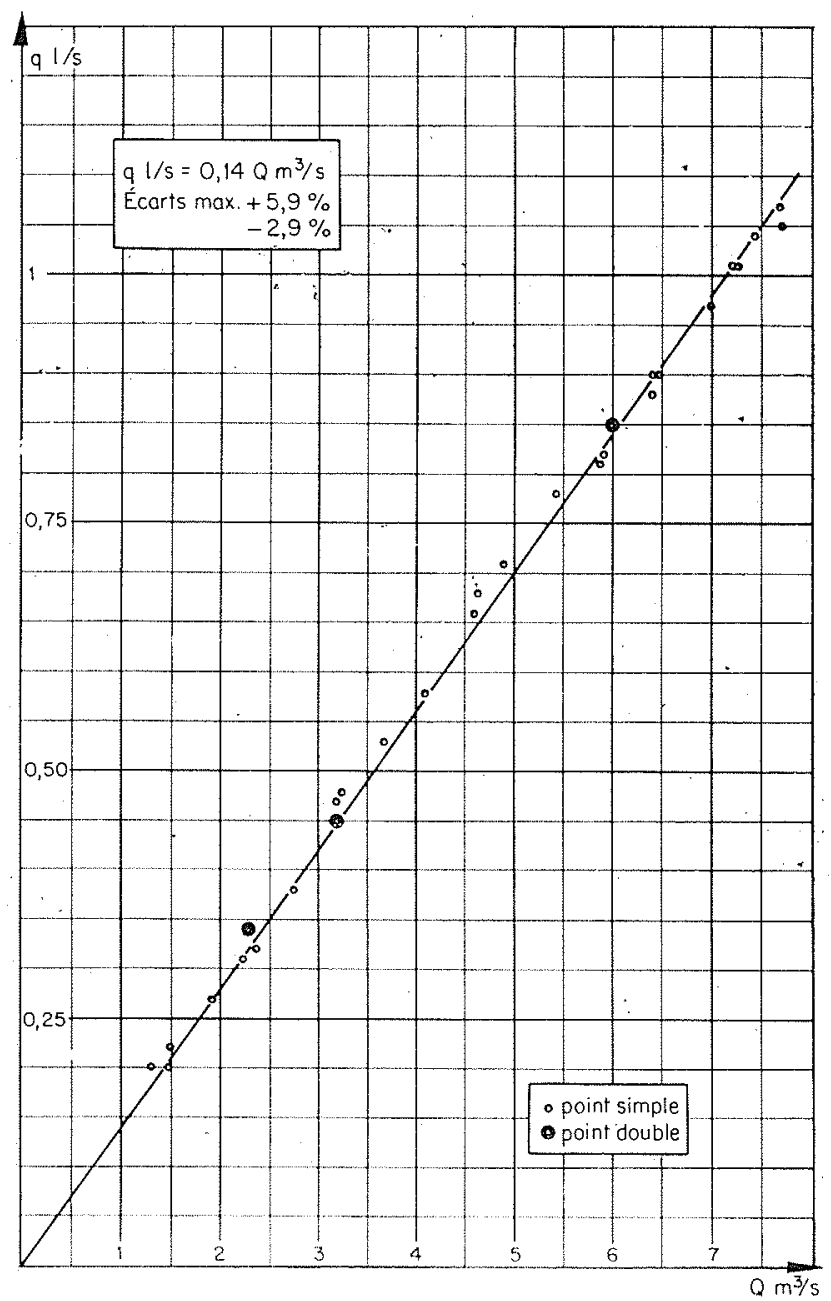

Fic. 9

Etalonnage de la dérivation par voie piẻzométrique.

\section{III. - RESULTATS - PRECISION}

Comme nous l'avons déjà écrit, un débitmètre de ce type a été installé à l'usine de La Bridoire en Savoie. Un fonctionnement continu de six mois nous a donné entière satisfaction. L'étalonnage est délicat si on veut obtenir une bonne précision, mais se conserve. Ce débitmètre répond très bien aux faibles variations de débit, et est insensible au pompage hydraulique qui se manifeste souvent dans la conduite forcée à l'amont des turbines.

Nous avons vu que la réponse du débitmètre était de la forme:

$$
\mathrm{Q}=\mathrm{A} q
$$

$\frac{\Delta \mathrm{Q}}{\mathrm{Q}}=\frac{\Delta \mathrm{A}}{\mathrm{A}}+\frac{\Delta q}{q}$

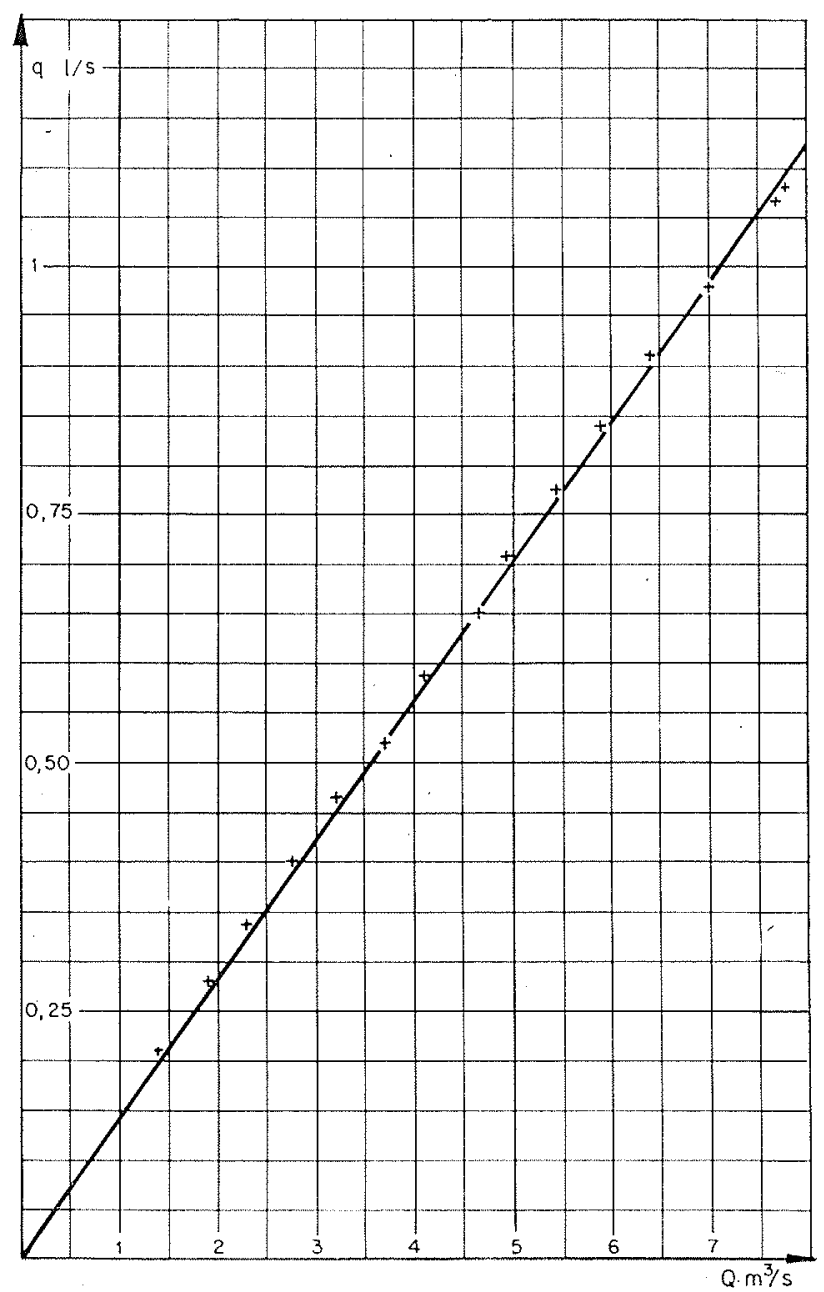

Fic. 10

Etalonnage de la dérivation par voie électromagnétique.

$\frac{\Delta Q}{Q}$ erreur sur le débit mesuré;

$\frac{\Delta \mathrm{A}}{\mathrm{A}}$ précision de l'étalonnage;

$\frac{\Delta q}{q}$ précision de l'appareil de mesure.

L'étalonnage du circuit dérivé en laboratoire nous a permis de constater que le débitmètre est sensible à une variation de débit dérivé de $5 \mathrm{~cm}^{3} / \mathrm{s}$. Cette variation s'inscrit sur l'enregistreur sous forme d'un décrochement d'environ un demi-millimètre.

$\frac{q}{\Delta q}=f(q)$ est une hyperbole d'équation $f(q)=\frac{5 \cdot 10^{-3}}{q^{1 / s}}$. 


\section{Précision de l'étalonnage :}

La vérification de la proportionnalité entre les débits étant faite, il suffit d'un point pour situer la droite de réponsc. Nous avons choisi le point correspondant au débit maximal pour avoir une précision meilleure.

$$
\begin{gathered}
\mathrm{A}=\frac{\mathrm{Q}_{m}}{q_{m}} \\
\frac{\Delta \mathrm{A}}{\mathrm{A}}=\frac{\Delta \mathrm{Q}_{m}}{\mathrm{Q}_{m}}+\frac{\Delta q_{m}}{q_{m}}
\end{gathered}
$$

Pour mesurer $Q_{m}$ nous avons utilisé la formule :

$$
\begin{gathered}
\mathrm{Q}_{m}{ }^{2}=\mathrm{K} h . \\
2 \frac{\Delta \mathrm{Q}_{m}}{\mathrm{Q}_{m}}=\frac{\Delta \mathrm{K}}{\mathrm{K}}+\frac{\Delta h}{h}
\end{gathered}
$$

$\frac{\Delta \mathrm{K}}{\mathrm{K}}$ est de l'ordre de $2 \%$. C'est l'erreur d'étalonnage du contrôle piézométrique. Elle est indépendante du débit.

$$
2 \frac{\Delta Q_{m}}{Q_{m}}=0,02+\frac{\Delta h}{h}
$$

$h$ était mesuré à l'aide d'un capteur à jauges extensométriques. La précision était de $4,2 \%$ pour le débit le plus faible, de l'ordre du millième pour les valeurs les plus élevées. Pour le point maximal, on pouvait négliger $(\Delta h / h)$ devant $2 \%$.

On a alors :

$\frac{\Delta Q_{m}}{Q_{m}}=0,01$

$\frac{\Delta \mathrm{A}}{\mathrm{A}}=0,01+\frac{5 \cdot 10^{-3}}{q^{\tau / s}}=0,01+\frac{5 \cdot 10^{-3}}{0,14 \mathrm{Q}^{\mathrm{m} 3 / \mathrm{s}}}$

$\frac{\Delta \mathrm{Q}}{\mathrm{Q}}=\frac{\Delta \mathrm{A}}{\mathrm{A}}+\frac{\Delta q}{q}=0,01+\frac{1}{14 \mathrm{Q}^{\mathrm{n} 3 / \mathrm{s}}}$

La courbe de précision en fonction du débit est représentée sur la figure 11.

\section{IV. - TOTALISATION DES DÉBITS}

Le système de totalisation est en cours d'élaboration. Son principe est très simple: il s'agit d'utiliser un moteur dont la vitesse de rotation soit proportionnelle à la tension qu'on lui applique. Ce moteur comprend deux enroulements. Le premier, qui sert à créer un champ magnétique, est monté en série avec le débitmètre. Il est done parcouru par le même courant régulé que les enroulements inducteurs du débitmètre. Le second est alimenté par la tension de sortie de l'amplificateur. C'est cette tension proportionnelle au signal qui commande la vitesse de rotation. Ce moteur entraine un compteur mécanique graduć en mètres cubes.

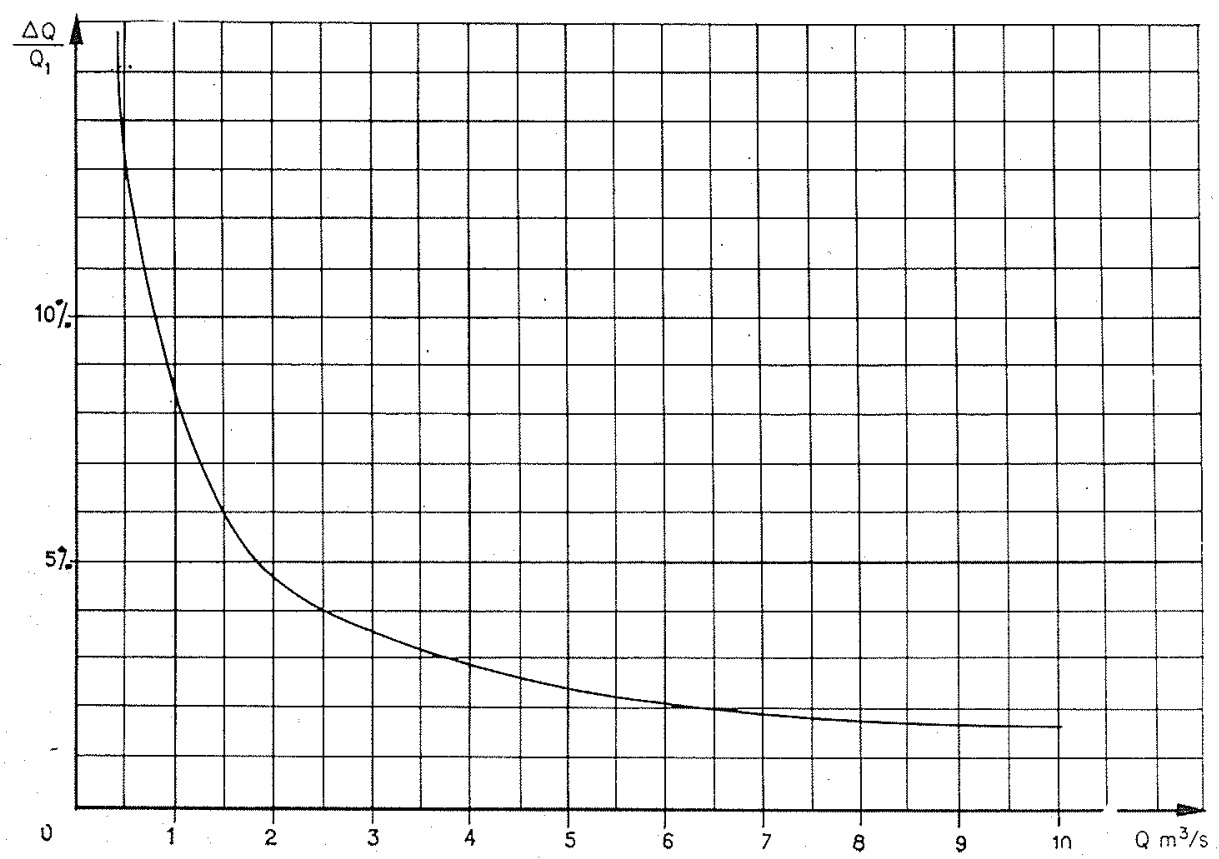

F16. 11

Courbe de précision, 


\section{C. - CONCLUSION}

Dans l'un et l'autre cas, nous avons utilisé pour mesurer le débit une différence de hauteur piézométrique; c'est l'exploitation de ce paramètre physique qui n'a pas été la mème. Il est donc logique d'envisager le domaine d'utilisation de chaque dispositif en fonction de la différence de pression disponible.

Avec un capteur piézométrique du type Serre-
Poncon, la précision est bonne pour des différences de pression supérieures à $1,50 \mathrm{~m}$.

Avec le dispositif électromagnétique, on conserve encore une bonne précision avec une différence de pression de l'ordre de $70 \mathrm{~cm}$.

Dans la zone où les deux méthodes peuvent ètre appliquées, l'expérience nous dira quelle est la plus fidèle, la fidélité restant le seul critère pourant justifier un choix.

\section{BIBLIOGRAPHIF}

[1] Brevet d'invention $n^{\circ} 1163940$, class, internationale E $303-$ GO. $1 \mathrm{f}$.

[2] Faraday. - Bakerian Lecture Diary (12-14 janvier 1832).

[3] Kor.ns. - Journal of Applied Physics, vol. 15 (février 1944); Review of Scient. Inst. (mai 1945).
[4] Thunlemaxn, - Helv. Phys. Aeta, no 5 et 6 (1941).

[5] Rimenuenas et Herman. - Mesure électromagnétique des vitesses dans les liquides. La Houille Blanche, $n^{\circ}$ spécial B (1954).

D I S C U S S I O N

président: M. Duffaut

M. le Président remercie M. Blanc-Fínaud pour l'exposé de cette communication et onvre la discussion.

Tout en estimant que la solution proposée par M. Branc-Fónaud est probablement la meilleure dans la cas considéré, M. Remeneras remarque qu'il est possible de construire un débitmètre électromagnétique pour conduite d'assez grand damètre sans sastreindre à mesurer le débit dans une dérivation shuntant une certaine longueur de ladite conduite.

La méthode utilisée notamment sur la conduite de refoulement (de l'ordre de $1 \mathrm{~m}$ de diamètre) de certaines dragues suceuses consiste à créer dans l'ensemble d'une section droite du tuyau ci-dessus un champ magnétique alternatif bipolaire; ce champ, qui est loin d'etre uniforme, est créé par deux enroulements extérieurs à la conduite; les forces électromotrices mesurées sur un diamètre perpendiculaire à l'axe du champ magnétique (moyennant certaines conditions assez peu restrictives en pratique), sont proportionnelles à la vitesse moyenne de la mixture d'eau et de sable traversant la conduite. Le système doit être, évidemment, étalonné.

On a proposé de mesurer de la même façon le débit d'une galerie d'amenée (section circulaire) d'usine hydroélectrique en disposant les barres de cuivre de l'enroulement inducteur (alimenté par un transformateur à très basse tension) dans le revêtement en béton de ladite galerie.

Les possibilités de ces systèmes sont limitées par la nécessité d'obtenir après filtrage un rapport suffisant entre le niveau de f.ém, signal (proportionnelle au débit) et celui des parasites, a priori d'autant plus importante que la section par le champ inducteur est étendue. C'est là un problème d'électronique qui est d'autant plus facile à résoudre que le champ inducteur est fort et son emprise réduite.

M. le Président remercie M. Remenieras de son intervention.

M. Caseau ajoute que, dans le cas d'un écoulement à filets parallèles, la possibilité de mesurer le débit par une méthode électromagnétique repose sur une équation du type:

$$
\int\left(\vec{B}_{0}^{2}\right) u d \tau=\Phi_{(O)} Y B_{0} d l
$$

C'est la première fois que ce thérème a été démontré. Faraday l'avait pressenti il $y$ a une centaine d'années. La démonstration en sera donnée dans la thèse de M. Blang-Friratio.

D'autre part, Thurleman a démontré que, dans le cas de conduit circulaire ef d'écoulement de révolution soumis à un champ uniforme, ce théorème s'étendait, non pas à l'intégrale du potentiel sur la courbe, mais à la différence de potentiel entre deux points. Ce théorème est indépendant de la répartition des vitesses.

Donc, on ne pourra connaitre, au mieux, que $\int\left(\mathrm{B}_{0}{ }^{2}\right) u d \sigma$ : d'où, si $B_{0}$ est non uniforme, la mesure dépendra $d u$ profil de vitesse.

La difficulté qu'il $\mathrm{y}$ a à créer un champ Bo uniforme conduit donc à utiliser des conduits de petites dimensions.

M. ReMENmiras dit que si l'on procède à l'étalonnage dans les conditions d'emploi, il suffit que le champ magnétique et le profil des vitesses restent constants dans le temps.

M. Caseau précise que, dans le cas où le champ Bo est uniforme, il n'y a pas interaction entre le champ et le profil des vitesses; on peut alors se passer d'étalonnage et on obtient une réponse linéaire. Dans le cas contraire, l'étalonnage devient nécessaire et ne sera valable que s'il existe une relation biunivoque entre le nombre de Reynolds et le profil des vitesses de l'écoulement. Quant à la courbe d'étalonnage, on ne peut pas en présumer.

M. Remevirisas pense que l'action des courants induits dans l'eau est négligeable et que, si l'on place le débitmètre électromagnétique à une distance raisonnable de la sortie de la pompe de la drague, on doit obtenir des résultats industriellement valables.

M. Branc-Frrayd pense que, dans le cas exposé par M. Remenreras, on peut utiliser une méthode d'impulsions de courant traversant l'enroulement inducteur; cette méthode permettrait d'avoir un champ plus grand, 
done d'augmenter le rapport: signal sur bruit de fond, et d'avoir des mesures plus précises.

M. ANDnÉ pense que dans le cas de la mesure des débits par dispositifs placés en dérivation, Ia D.T.G. a pu constater que la loi $Q=f(q)$ du débit de la conduite principale en fonction du débit dans la dérivation n'était linéaire que pour des valeurs suffisantes du nombre de Reynolds. Pour les faibles débits, marche $\grave{a ̀ ~ v i d e ~ d ' u n e ~ u s i n e ~ h y d r o e ́ l e c t r i q u e ~ p a r ~ e x e m p l e, ~ l e ~}$ nombre de Reynolds relatif à la dérivation peut être inférieur à 10000 , alors qu'il se maintient bien audessus de 100000 pour la conduite principale.

M. ANdRÉ rappelle que M. Branc-Fŕnaud a indiqué très justement que l'on avait intérêt à créer une perte de charge localisée dans la dérivation : pour que cette perte de charge soit stable dans le temps et quadratique, il faut cependant veiller à ce que l'écoulement corresponde à des nombres de Reynolds suffisants malgré cette perte de charge, ce qui conduit à choisir des prises d'eau et des conduites en dérivation d'un diamètre suffisant.

M. BLANC-FÉRAud confirme que le plus important est d'avoir une perte de charge quadratique.

M. Blanc-Féraud précise d'autre part qu'il est pratiquement impossible de faire un calcul théorique du débit dérivé en fonction du débit principal, car l'on ne sait pas du tout ce qui se passe. Dans la dérivation, le profil des vitesses peut être modifié, mais cela n'a pas d'importance, puisque le débitmètre électromagnétique mesure la vitesse moyenne quelle que soit la répartition des vitesses. Si l'ensemble de la dérivation se comporte comme une perte de charge quadratique, même si le régime est alors laminaire dans la section de mesure, le principe de la méthode reste valable. Il faudra tout au plus augmenter la sensibilité de l'appareillage de mesure.

M. Gabaudan ajoute que le coefficient désigné pa: $\alpha$, caractérisant la répartition des vitesses dans les sections de vamne de part et d'autre de l'organe déprimogène utilisé pour la mesure, peut être influencé par les conditions d'écoulement à l'aval; en particulier, le régime do fonetionnement de la turbine et a fortiori la position de la vanne de garde de celle-ci peuvent avoir une incidence sensible. Il peut en résulter un détarage de l'appareil.

M. Blanc-FÉRaud pense que cela est possible, bien gu'il ne l'ait pas constaté. Dans le cas de La Bridoire, le dispositif électromagnétique restait assez stable, et paraissait être indépendant du pompage dû probablement $\dot{a}$ la turbine installée à l'aval, et que l'on observait par contre avec Ie dispositif piézométrique. Il est évident que le dispositif électromagnétique répond à une vitesse et non à une pression. Pour se libérer de l'inconvénient dont parle M. GABAUdan, il faudrait utiliser l'énergie globale de la section au lieu de n'en prélever qu'une petite partie, e'est-í-dire mesurer le débit global.

M. le Président a eu l'occasion de voir employer par la Région d'Equipement Hydraulique «Garonne d'E.D.F., sur la conduite forcée de la chute de l'Hospitalet, à Lanoux, un dispositif qui utilise un venturi, mais avec mesure de précision des différences de niveau par une balance hydrostatique du type Rittmeyer de Suisse.

M. Branc-Fŕraud a-t-il une idée de la précision de ce type de mesure?

M. Branc-Féraud n'a pas idée de la précision mais dit qu'à l'usine de Génissiat, pour mesurer le débit, la Compagnie des Compteurs avait installé, dans la conduite forcée d'environ $4 \mathrm{~m}$, un venturi suivi de manomètre à membrane du type décrit dans sa communication. Du fait du pompage hydraulique, le contact des manomètres s'était détérioré très rapidement.

La mise en cuvre de balances hydrostatiques nécessite un asservissement assez compliqué et plus difficile que celui utilisé dans la méthode électromagnétique, mais sa précision est peut-être meilleure.

M. WoLF précise que l'utilisation de ces appareils nécessite une perte de charge importante de l'ordre de $10 \mathrm{~m}$ d'eau, tandis que les manomètres à transmission électrique utilisés ici n'exigent environ qu'un mètre d'eau de perte de charge.

M. le Président demande, étant donné que l'on produit une force électromotrice à partir d'un champ magnétique et d'un écoulement d'eau, si l'on ne pourrait pas remplacer les turbines et les alternateurs par des enroulements autour des conduites forcées, ce à quoi M. BLancFéraud répond que M. Remenieras l'a déjà envisagé, mais que le rendement est déplorable.

M. REMENiERas résume comme suit les raisons qui limitent les possibilités pratiques d'un tel système (1):

La résistivité du cuivre industriel étant de 1,6 microhm par $\mathrm{cm} . \mathrm{cm}^{3}$, celle des solutions aqueuses pour la concentration donnant le maximum de conductibilité est de plusieurs ohms par $\mathrm{cm} . \mathrm{cm}^{2}$ (1,84 pour $\mathrm{KOH}, 4,66$ pour $\mathrm{NaCl}, 5000$ à $10^{\circ}$ pour l'eau ordinaire). Il en résulte qu'à force électromotrice égale, l'intensité des courants électriques dans les meilleures solutions aqueuses sera un million de fois plus faible que dans le cuivre; les forces, les couples et les pertes ollmiques seront dans le même rapport. En outre, les vitesses des fluides pratiquement réalisables sont généralement inférieures aux vitesses périphériques des générateurs électriques tournants (sauf s'il s'agit de gaz ionisés).

Dans le domaine des liquides, seul le sodium (résistivité de l'ordre de 10 microhms $/ \mathrm{cm}^{-\mathrm{cm}^{2}}$ ) et certains de scs alliages présentent une résistivité suffisamment faible pour permettre certaines réalisations industrielles telles que les pompes électromagnétiques à métaux liquides utilisées pour la circulation de l'alliage de sodium assurant les transferts de chaleur dans certains réacteurs nucléaires; leur rendement est de l'ordre de 40 à $50 \%$.

Ces pompes (2) sont de divers genres et constituent l'équivalent des divers types de moteurs électriques classiques; comme ces derniers, elles sont «réversibles». C'est ainsi que sí, dans une pompe asynchrone triphasée à champ tournant (ou glissant), on fait passer sous l'action d'une pression extérieure un écoulement de lluide conducteur à une vitesse supérieure à celle du «synchronisme», on obtient une génératrice électrique hypersynchrone qui transfère au réseau général, sous forme électrique, une partie de l'énergie de la veine fluide qui la traverse : ceci est réalisé sans aucun contact matériel entre le fluide et les parties électriques de la machine et sous une tension qui est celle de l'enroulement du moteur asynchrone (laquelle peut atteindre plusicurs milliers de volts).

Actuellement, le seul fluide conducteur acceptable serait le sodium ou l'un de ses alliages : demain, les nombreuses recherches de magnétohydrodynamique actuellement en cours permettront peut-être d'utiliser des flammes et plasmas à très haute températuro et fortement ionisés.

M. le Président remercie M. Blanc-Fḱraud pour sa communication et toutes les personnes ayant pris part à la discussion.

(1) G. Ramenieras. - Sur la possibilité de transformer directement en énergie électrique une partie de l'énergie d'une veine fluide, $L a$ Houlle Blanche, $\mathrm{n}^{\circ}$ spécial "A " (1948).

(2) C. Hermant. - Pompes à métaux liquides (Mémoires et Travaux de la Sociéte Hudrotechnique de France (1957), suppl. au volume $I, C . R$. des séances du C.T. organisées le 15-11-1956). 